\title{
DIFFERENT APPROACHES TO CRYSTALLIZATION OF MEMBRANE PROTEINS
}

\author{
${ }^{1}$ Prakash G. Doiphode and ${ }^{2}$ Vivek Pratap Singh \\ ${ }^{1}$ Department of Chemistry, University of Louisville, Louisville, Kentucky \\ ${ }^{2}$ Department of Pharmacology and Cell Biophysics, \\ University of Cincinnati, College of Medicine, Cincinnati, Ohio
}

Received 2012-05-22, Revised 2012-06-01; Accepted 2012-12-18

\begin{abstract}
Crystallography is more like an art than science. Crystallizing membrane proteins are a big challenge; membrane proteins are present in the cell membrane and serve as cell support. The most important feature of membrane protein is that it contains both hydrophobic and hydrophilic regions on its surface. They are generally much more difficult to study than soluble proteins. The problem becomes more difficult when trying to obtain crystals to determine the high resolution structures of membrane proteins. We want to utilize this opportunity to briefly examine various approaches for crystallization of membrane proteins. The important factors for determining the success of crystallization experiments for membrane proteins lies in the purification, preparation of membrane samples, the environment in which the crystals are grown and the technique used to grow the crystals. All the X-ray structures of membrane protein are grown from preparations of detergents by different methods developed to crystallize. In this review different techniques for the crystallization of membrane proteins are being described. The cubic phase method also known as in meso method is discussed along with other methods to understand about the crystallization of membrane proteins, its general applicability, salt, detergent and screening effects on crystallization. Low volumes as nano-liter of samples can be used for crystallization. The effects of different detergents on the crystallization of membrane protein, as well as the use of surfactants like polyoxyethylene. Approach based on the detergent complexation to prove the ability of cyclodextrins to remove detergent from ternary mixtures in order to get $2 \mathrm{D}$ crystals. Crystallization of membrane proteins using non-ionic surfactants as well as Lipidic sponge phase and with swollen lipidic mesophases is discussed to better understand the crystallization of membrane proteins.
\end{abstract}

Keywords: Crystallization, Membrane Proteins

\section{INTRODUCTION}

Membrane proteins are those that simply exist in the cell membrane and are essential for cellular life. They contribute too many processes where by the cells interact with the environment and generate energy and maintain the cellular structure (Wiener, 2004). They serve as both active and passive channels for ions and chemicals. They perform many important functions like nerve impulse transmission and transport for into and out of the cell and are also important targets for many drugs (Chiu et al.,
2000). These factors make them very important from the study point of view and crystallography is the most reliable way to explore membrane protein structurefunction relationship in atomic detail (Chiu et al., 2000). To perform crystallography a good quality crystals is essential which are not readily produce in case of membrane proteins, thus providing a big challenge for the crystallographers. One important feature of membrane proteins is that it has both the hydrophobic and hydrophilic regions on the surface; that allows the protein to have a hydrophobic region formed by the lipid

Corresponding Author: Vivek Pratap Singh, Department of Pharmacology and Cell Biophysics, University of Cincinnati, College of Medicine, Cincinnati, Ohio, Tel: 513-558-2327 
double layer which makes most of the membrane while still to have a stable interface with the aqueous layer on either side of the membrane (Caffrey, 2003). Due to the structural restrictions of membrane proteins their structural possibilities have become more limited.

Most of the crystallization technique uses aqueous solvent for crystallization and the membrane proteins get easily denatured in this environment and loose their structure. Some common features of membrane proteins include the $\alpha$-helices, $\beta$-barrel and are in the hydrophilic residues of amino acids. In the early 1980s the detergentbased micelles was rationally designed to solubilize membrane proteins and than the first membrane protein crystal was crystallized, it was of a photosynthetic reaction centre in the year 1984, the German group who crystallizes the membrane protein was awarded with a Noble Prize for their work (Caffrey, 2003). Membrane protein crystal structures are being produced and the work is going on to crystallize membrane proteins to study their X-ray structures in detail. The methods used for the crystallization of membrane proteins mainly involve the in serfo method and the cubic-phase method.

The in serfo method was the first successful method to crystallize membrane proteins and a good number of crystals were crystallized with this method. The in serfo is a liquid solution based and can be implemented for vapor diffusion for soluble proteins. In the late 90 s a new method was developed which is known as the cubic-phase method or the in meso method, the cubic-phase method is itself constituted of a lipid bilayer and so the lipid is very comfortable here, this method has gained popularity in a very short period of time and reasonable number of membrane proteins have been crystallize successfully using the in meso method (Wiener, 2004). The other two methods which are very recent, are the bicellie method and the vesicle-fusion method, they are still in their infancy stage as both the techniques are pretty new but both the methods have produced one crystal of membrane protein. Thus so far the most successful method is the cubic- phase or the in meso method. In this review new approaches with the in meso methods along with few other approaches have been studied so as to understand the different aspects of crystallizing membrane proteins and try to overcome the drawbacks which interfere with the formation of crystals (Cherezov et al., 2004).

\subsection{Approaches for Crystallization of Membrane Proteins}

\subsection{First Approach}

The first approach is the controlled 2D crystallization of membrane proteins by using methyl- $\beta$ - cyclodextrin (Signorell et al., 2007). This method gives detailed information about the ability of cyclodextrins to remove detergents from ternary mixtures like lipids and proteins in order to get high resolution 2D crystals of membrane proteins. It has been tested on OmpF, which is a $\beta$-barrel protein and also with SoPIP2; 1 which is a $\alpha$-helical protein. The crystals produced with this approach had better resolution when compared with the previous crystallization reports and so this approach by cubic-phase technique is found to improve the resolution of the crystal structures of membrane proteins. The cubic-phase method requires large amount of lipid and proteins, to find enough purified membrane protein is a big task, also the crystallization is performed in small tubes where visual inspection is difficult and the process of high resolution structure determination is very tedious and unpredictable.

\subsection{Second Approach}

The second cubic-phase approach uses nano-volume which has excellent optical properties. These new crystallization plates offer significant advantages for the in meso crystallization of the membrane proteins. The plates are made from the glass microscope slides and cover slips which are cost effective. The glass used for these plates had excellent optical activity which helped to view the process of crystallization through the glass windows. The plate uses a silicon rubber gasket which forms the walls of the wells. With the use of nano-volumes the crystal formation is seen to be very fast and with these method crystals of bacteriorhodospin grows successfully (Cherezov and Caffrey, 2003; Kubicek et al., 2012).

\subsection{Third Approach}

The third approach makes use of the liquid analogue of the cubic phase, the sponge phase. The sponge phase facilitates considerable increase in the size of the crystals formed. Since the cubic-phase is curved with smaller inner pore that makes it difficult for the host membrane protein with larger hydrophilic domains. In order to make it easier for the protein to diffuse in the lipid layer, it can be flattened out by the use of solvents such as polyethylene glycol, propylene glycol or dimethyl sulfoxide to the $\mathrm{MO} /$ water system in order to create a sponge phase. This bicontinuous phase is a transparent liquid with an inner structure which will look like a melted cubic phase and will have less curved lipid layer and two to three times bigger aqueous pores. In this approach the reaction center from the Rhodobactor sphaeroids can be crystallized by using a sponge phase with an ordinary hanging drop experiment. The 
crystallization of the membrane protein from the sponge phase can be confirmed by visual inspection with Small Angle X-ray Scattering (SAXS) and also with NMR measurements. This approach of producing crystals is found to be very advantageous as the vapor phase diffusion crystallization can be applied directly. As compared to the earlier lipidic cubic-phase, the mobile ubiquinone is more refined. The different advantages of the sponge phase make this an important approach for the crystallization of membrane proteins (Wadsten et al., 2006).

\subsection{Fourth Approach}

The fourth approach of cubic-phase for crystallization of membrane proteins uses additives that swell the cubic phase. Normally proteins move in the bilayer in a lateral manner in the mesophase which gives effective nucleation and crystal growth. If the protein's extra membrane is large relative to the dimensions of the layers in which it connects, than the mobility of the proteins will be restricted which will result in a decrease in the growth rate of the crystals or the growth can stop completely. Thus with the in meso method the size could be increased to include large membrane proteins and so the crystallization of the smaller proteins would get an acceleration in the cubic phase with larger aqueous channels, this can happen by inducing a swelling of the meso phase while maintaining the properties of a continuous lipid bilayer of the membrane protein. The cubic-phase can be swollen by the use of small water soluble organic molecules and inorganic salts, with the $\mathrm{X}$-ray diffraction; the mesophase swelling was identified and is seen to be a function of additive concentration in the aqueous medium. The crystal structures formed from this method are comparable to the detergent-grown crystals with a little difference in crystal packaging (Cherezov and Caffrey, 2006).

\subsection{Fifth Approach}

The last approach for crystallization of membrane proteins was making use of non-ionic surfactants which are generally n-alkyl polyoxyethylenes. It is seen that a variety of protein precipitating agents like polyethylene glycol, ammonium sulfate and methylene pentate diol are added to the protein solution to start crystallization and there is very less understanding of the process involved. It can be a common place for the two water soluble polymers to have a separate solution phase at specific polymer concentration. In case of membrane protein there is a problem in keeping them in the solution phase after solublization from the membrane, at this time detergents are used to solubilize the protein but at times it acts against the desired solubility and is not good for crystallization, so non-ionic surfactants are used for this process. These non-ionic surfactants are very mild and have a very less effect on the protein conformation. These surfactants form a separate aqueous phase with the water soluble polymers, since the surfactants micelle form a second polymer species which promotes phase separation, it is possible to use non-ionic surfactants that can be used in place of PEG. With use of non-ionic surfactants membrane proteins can be solublized and crystals can be produced (Liu and Cherezov, 2011).

\subsection{Cubic Phase with Cyclodextrins Approach}

Cyclodextrins can form complex with any kind of molecule and is an advantage over the dialysis methods. During crystallization the nature of the detergent used, amount of detergent removed as well as the rate at which it is removed affects the size and quality of the crystals. These mild detergents are widely used for the purification of large and sensitive complexes. The cyclodextrin approach produced OmpF crystals of different qualities depending on the reconstitution time. For $2 \mathrm{~h}$ reconstitution gives a low quality crystal and when the reconstitution is longer from 12 to $144 \mathrm{~h}$ it results in average or bigger size crystals. The crystals are comparable to the one grown with dialysis method. The phospolipase helps to improve the packaging of the crystals. With SoPIP2; 1 the reconstitution over $2 \mathrm{~h}$ shows smaller crystals and with $144 \mathrm{~h}$ reconstitution shows larger crystals. The quality of the crystals obtained by this method is comparable and can give a spot diffraction up to a resolution of $4 \AA$. The unwashed crystals show additional diffraction spots and can be arranged in six fold symmetry. The problem of evaporation can be solved by using micro-droplet pipettes to dispense cyclodextrins along with calculated volumes of water. It is seen that during longer duration the crystals are bigger in size and of good quality. With cyclodextrins small amount of volume is required and no additional buffer is required. Thus cyclodextrin can be used with those compounds which are very difficult to produce (Signorell et al., 2007).

\subsection{Nano-volume plates with optical properties}

In the nano-volume approach the beneficial part is its compatibility to work with very small volumes as with the manual dispensing method only $50 \mathrm{~nL}$ of lipid/ protein mesophase is used that corresponds to 200ng of protein per crystallization trial. Crystallization can be studied on samples which had $<5 \mathrm{~nL}$ of mesophase and $<20$ ng of protein. Excellent quality crystals can be 
produced with nanoliter volume samples. A minimum of $3 \mathrm{~nL}$ volume is needed to produce bacteriorhodospin crystals. The manual delivery of such small volumes did not produce the same consistent results, so it requires the whole process to be automated to give consistent, results. By using the glass plates with the in meso method small volumes can support faster growth of crystals for membrane proteins. Even with bolus, if smaller bolus used the sooner the crystals can appear. Crystals grown when sandwiched as a thin layer between the glass plates, helps for light microscope viewing of the crystallization process. These plates can also be used with a modification in the bicelle method. With the new modified technique even picoliterscale crystallization is made possible (Cherezov and Caffrey, 2003; Ujwal and Bowie, 2011).

\subsection{Lipidic Sponge Phase Approach}

With the Lipidic cubic phase (LCP) crystallization of membrane proteins MO (monoolein) is mixed with the protein in the aqueous solution and the resulting cubicphase is placed in contact with the precipitant solution, the precipitant solution leads to phase transformation in the MO/water system. The setups appeared to liquefy indicating that there is a phase transformation, these phase transformations improves the crystals quality. The simplest method to understand the crystallization process from LCP is to add a crystal violet to the cubic phase without protein; it gets accumulated at the detergent/protein interface. After $20 \mathrm{~h}$ of precipitate addition the semi-solid cubic-phase is gone resulting in a two liquid phase, thus most of the MO is moved to the upper phase which was also can be confirmed by NMR. Small Angle X-ray Scattering (SAXS) can be used to examine the phase transformation of the LPC but without protein and can be measured for every 30mins for a period of $16 \mathrm{~h}$. The X-ray scattering shows cubic phase with Pn $3 \mathrm{~m}$ symmetry and after $5 \mathrm{~h}$ additional diffraction peak is observed with space symmetry Ia3d. $8 \mathrm{~h}$ later Pn3m pattern disappeared and after $10 \mathrm{~h}$ Ia3d disappeared and a diffusion Brags peak appeared, it is interpreted that the Ia3d pattern can be due to the cubic-phase while the Brags peak was due to the formation of the sponge phase. Since the sponge phase is in the liquid form, it can be directly used in the vapor diffusion or hanging drop method. The method mixes four parts of sponge phase with one part of concentrated protein solution, crystals were seen to form after one week and these crystals structures showed a different appearance than that grown from LPC and were larger in size (Wadsten et al., 2006; Johansson et al., 2009; Cherezov, 2011).

\subsection{Using Additives to Swell the Meso-Phase}

The swollen lipid mesophase, water-soluble additives cause the cubic-phase to swell. The swelling increases the lattice parameter of the cubic-phase with an increase in the aqueous solution that supports liquid solution phase formation and stability. A larger lattice is due to the increase in the aqueous channel diameter. The phase diagram for monoolein/water indicates that the cubic Pn3m phase exists in equilibrium with an excess aqueous phase. The additives examined causes the cubic phase to swell at relatively low concentration and beyond a certain limit the cubic Pn $3 \mathrm{~m}$ phase is transformed in to a phase which is similar to a sponge form. The sponge like phase continues to swell and thus it gets converted in to lamellar liquid crystal phase. Urea and $\mathrm{GndCl}$ both show swelling for the cubic-Pn3m phase, but in case of arginine, swelling of the cubic-phase formed can be from Im $3 m$ type. Thus apart from arginine all other additives can cause the cubic-Pn $3 \mathrm{~m}$ phase to swell. The sponge phase can be considered as a swollen, disordered cubicphase; it is characterized by the low range $\mathrm{X}$-ray scattering and is isotropic like the cubic-phase but is also stiff and viscous. The crystal seen with membrane proteins by the in meso method growth was seen in the concentration range where the cubic-phase is swollen and converts in to the sponge like form. $\mathrm{GndCl}$ can also causes the swelling of the cubic-phase, but doesn't seen to support the crystallization of protein, since $\mathrm{GndCl}$ is a powerful denaturant which might have avoided the crystal formation. With sucrose and glycerol the cubic phase was seen to shrink in size. The structures of the two membrane proteins LH2 and BtuB grown from the meso phase were of diffraction quality and useful for structure determination. The LH2 is a large protein complex and its structure was even further investigated and it showed two concentric rings of transmembranal peptide subunits with the diameter of $36 \AA$ and $68 \AA$. The inner and outer ring contained nine $\alpha$ and nine $\beta$ peptides respectively (Cherezov et al., 2006; Liu and Cherezov, 2011; Hofer et al., 2010).

\subsection{Non-Ionic Surfactants Approach}

Uses of non-ionic surfactants in the cubic phase method shows the crystal growth to be more rapidly at lower $\mathrm{pH}$ than higher $\mathrm{pH}$ as seen with HEWL crystallization. Crystallization shows few membrane proteins but none of the membrane protein produced crystals which can be suitable for X-ray diffraction in contrast crystals were looks more suitable for soluble proteins (Mustafa et al., 1998). 


\subsection{Conclusion and Perspective}

Cyclodextrins can be used to improve the quality of the crystals along with phospholipase. The quality of OmpF as well as SoPIP2; 1 crystals is improved. Cyclodextrins controls the rate of detergent removal with accuracy and is not depend on the CMC of the detergent and can work with small volumes. Due to the optical properties successful administration of the process is possible. Superior quality crystals were seen to be produced by this method. In sponge phase it is seen that with RCsph, the LCP goes from Pn3m to Ia3d to $\mathrm{L}_{3}$ and finally to the $\mathrm{L}_{\alpha}$. The main purpose here was to understand the phase transformation occurring during the crystallization experiment. With the swollen lipidic mesophase the tendency of the additives to affect mesophase swelling shows their ability to support in meso crystallization of membrane proteins. The swelling of cubic phase was found to be most beneficial in case of proteins with large extramembranal domains. The nonionic surfactants looked to be more suitable for crystallization of membrane proteins because these surfactants were mild and were suitable for crystallization as the membrane proteins are more sensitive to denaturation. The lipid meso-phase and many other approaches are now developed with high robotic methods and can give consistent results, with nanoliter and picoliter-scale. Most of the approaches discussed in this review are unique and had shown improved results for crystals of membrane proteins, except the non-ionic surfactants. This review gives an understanding of the crystallization process which will help to overcome the drawbacks of crystallizing membrane proteins.

\section{REFERENCES}

Caffrey, M., 2003. Membrane protein crystallization. J. Stru. Biol., 142: 108-132. DOI: 10.1016/S10478477(03)00043-1

Cherezov, V and M. Caffrey, 2003. Nano-volume plates with excellent optical properties for fast, inexpensive crystallization screening of membrane proteins. J. Applied Cryst., 36: 1372-1377 DOI: 10.1107/S002188980301906X

Cherezov, V and M. Caffrey, 2006. Picolitre-scale crystallization of membrane proteins. J. Applied $\begin{array}{lll}\text { Cryst., } & 39 \text { : } & \text { 604-606. }\end{array}$ 10.1107/S0021889806022953

Cherezov, V., 2011. Lipidic cubic phase technologies for membrane protein structural studies. Curr. Opin. Stru. Biol., 21: 559-566. DOI: 10.1016/j.sbi.2011.06.007
Cherezov, V., A. Peddi, L. Muthusubramaniam Y.F. Zheng and M. Caffrey, 2004. A robotic system for crystallizing membrane and soluble proteins in lipidic mesophase. Acta Cryst., D60: 1795-1807. DOI: $10.1107 / \mathrm{S} 0907444904019109$

Cherezov, V., J. Clogston, M.Z. Papiz and M. Caffrey, 2006. Room to move: Crystallizing membrane proteins in swollen lipidic mesophases. J. Mol. Biol., 357: 1605-1618. DOI: 10.1016/j.jmb.2006.01.049

Chiu, M.L., P. Nollert, M.C. Loewen, H. Belrhali, E. Pebay-Peyroula et al., 2000. Crystallization in cubo: General applicability to membrane proteins. Acta Cryst., 56: 781-784. DOI: 10.1107/S0907444900004716

Hofer, N., D. Aragao and M. Caffrey, 2010. Crystallizing Transmembrane Peptides in Lipidic Mesophases. Biophys. J., 99: L23-L25. DOI: 10.1016/j.bpj.2010.05.011

Johansson, L.C., A.B. Wohri, G. Katona, S. Engstrom and R. Neutze, 2009. Membrane protein crystallization from lipidic phases. Curr. Opin. Stru. Biol., 19: 372-378. DOI: 10.1016/j.sbi.2009.05.006

Kubicek, J., R. Schlesinger, C. Baeken, G. Büldt and F. Schäfer, 2012. Controlled in meso phase crystallization-a method for the structural investigation of membrane proteins. PLoS ONE., 7: e35458-e35458. 10.1371/journal.pone. 0035458

Liu, W. and V. Cherezov, 2011. Crystallization of membrane proteins in lipidic mesophases. J. Vis. Exp. DOI: 10.3791/2501

Mustafa, A.O., J.P. Derrick, G.J. Tiddy and R.C. Ford, 1998. A novel approach for the crystallization of soluble proteins using non-ionic surfactants. Acta Cryst. D. Biol. Cryst., 54: 154-158. PMID: 9867432

Signorell, G.A., T.C. Kaufmann, W. Kukulski, A. Engel and H.W. Rémigy, 2007. Controlled 2D crystallization of membrane proteins using methyl$\beta$-cyclodextrin. J. Stru. Biol., 157: 321-328. DOI: 10.1016/j.jsb.2006.07.011

Ujwal, R and J. U. Bowie, 2011. Crystallizing membrane proteins using lipidic bicelles. Methods, 55: 337341. DOI: 10.1016/j.ymeth.2011.09.020

Wadsten, P., A.B. Wohri, A. Snijder, G. Katona, A.T. Gardiner et al., 2006. Lipidic sponge phase crystallization of membrane proteins. J. Mol. Biol., 364: 44-53. DOI: 10.1016/j.jmb.2006.06.043

Wiener, M.C, 2004. A pedestrian guide to membrane protein crystallization. Methods, 34: 364-372. DOI: 10.1016/j.ymeth.2004.03.025 